\title{
Nurturing growth with excellence: PMBP goes monthly in its Silver Jubilee year!
}

\author{
N. Raghuram ${ }^{1}$ Rana P. Singh ${ }^{2}$
}

Published online: 23 January 2020

(c) Prof. H.S. Srivastava Foundation for Science and Society 2020

This is indeed a very happy New Year for all of us involved with PMBP, as we celebrate the journal's Silver Jubilee with a new cover. This issue also marks the beginning of monthly publication of PMBP, a major milestone in our 25 years long journey. PMBP started as a modest halfyearly journal in 1995 and became a quarterly in its 12th year in 2006 in accordance with our growth plan (Editorial 2006). It took another 12 years to become a bimonthly in 2018 , but the subsequent growth of the journal has been rapid. After only 2 years as a bimonthly with 6 issues per year, we are doubling the frequency of publication as a monthly from January 2020. The 3.4 fold growth of inward submissions since 2015 and their improving quality has allowed us to double the total number of published articles (Fig. 1), even while we increased the rejection rate to over $80 \%$ to maintain quality and nudge the journal forward.

Since the last editorial on the growth performance of the journal (Raghuram, 2017), PMBP crossed another important milestone of 10,000 citations in Google Scholar and has reached 11,537 citations at the time of this writing (Fig. 2), with a h-index of 43 and i10 index of 363 (https:// scholar.google.co.in/citations?hl=en\&user=hLrG91UAAAA J\&view_op=list_works\&sortby=pubdate). The ISI impact factor at Clarivate Web of Science has grown steadily to 1.539 while its equivalent at Scopus (2 year impact) is currently at 1.618 (Fig. 3). The 4 year impact on Scopus had crossed 2 for the last two consecutive years, an indication of

N. Raghuram

raghuram@ipu.ac.in

1 School of Biotechnology, Guru Gobind Singh Indraprastha University, New Delhi 110078, India

2 Department of Environmental Sciences, Babasaheb Bhimrao Ambedkar University, Lucknow 226025, India

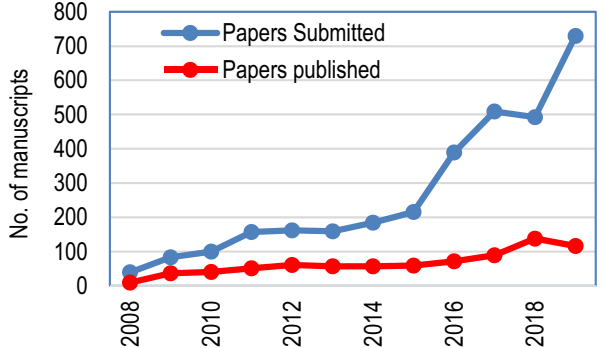

Fig. 1 Growth in the number of papers submitted and published

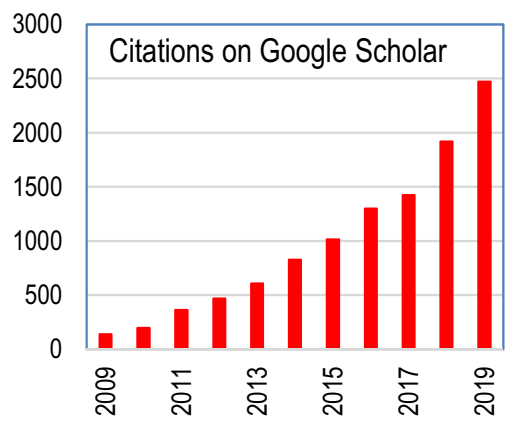

Fig. 2 Google scholar citations

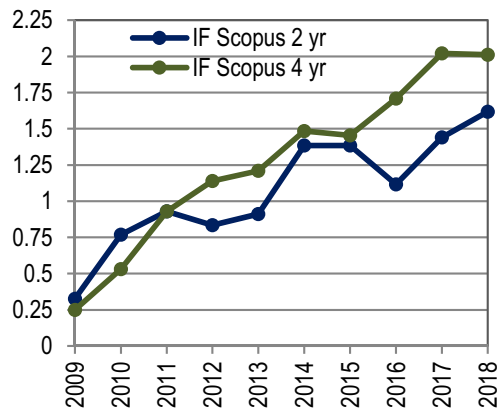

Fig. 3 Growth in impact factor (Scopus) 


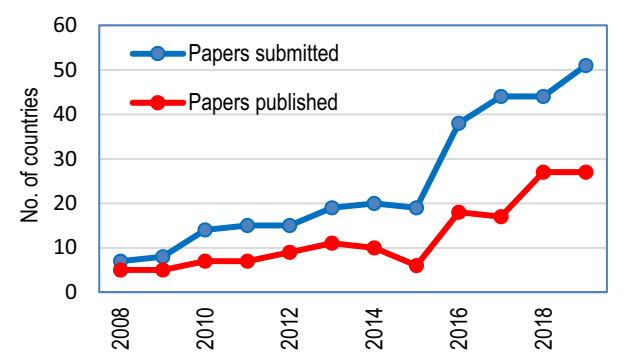

Fig. 4 Growth in the no. of countries contributing to PMBP (by the country of corresponding author)

the ISI impact factor it can achieve later this year or next year. We have a long way to go to meet our own targets of excellence in terms of impact factor, but even at the current level of performance, PMBP ranks 154th among 440 plant science journals around the world indexed by Scopus, somewhere between the top one-third and the top quartile.

We thank our authors, reviewers and editors for their contributions in our collective journey towards excellence. Our discerning readers may have already noticed the improvements in the journal's content. The geographical spread of incoming articles has improved, as has our footprint of published articles and citations from more countries than ever before (Fig. 4). Hundreds of new reviewers have been added from many countries and several handling editors have been added to improve the editorial flow in different sub-disciplines. Our consistent efforts to raise the bar in some areas like tissue culture and molecular markers have clearly helped in maintaining a more even distribution across different areas of plant functional biology. The thematic diversity of articles has also improved in accordance with the broad scope of PMBP to span all areas of plant biology.

It is very satisfying that this growth is exactly as envisioned many years ago (Editorial 2006), in accordance with our understanding of the growth dynamics of global plant science literature in the previous decades (Raghuram 2004). Further, as a vindication of our strong editorial commitment and measures taken to enforce publishing ethics (Raghuram 2017), we have not received any more complaints on publishing misconduct in PMBP in the last 2 years. Even a search at the Pubpeer site by the name of the journal did not produce any results at the time of this writing! We look forward to continue to strive for excellence on all fronts with the cooperation of all our stakeholders.

\section{References}

Editorial (2006) Physiol Mol Biol Plants 12(1):1

Raghuram N (2004) Indian publishing. Enduring the boom. Trends Plant Sci 9(1):9-12

Raghuram N (2017) The pleasure of excellence-led growth and the pain of enforcing publishing ethics: the experience of PMBP. Physiol Mol Biol Plants 23(1):1-3

Publisher's Note Springer Nature remains neutral with regard to jurisdictional claims in published maps and institutional affiliations. 